\title{
Health and Technology Magazine Editorial: Global CE Day 2021
}

\section{Tom Judd $^{1}$}

Published online: 25 October 2021

(c) IUPESM and Springer-Verlag GmbH Germany, part of Springer Nature 2021

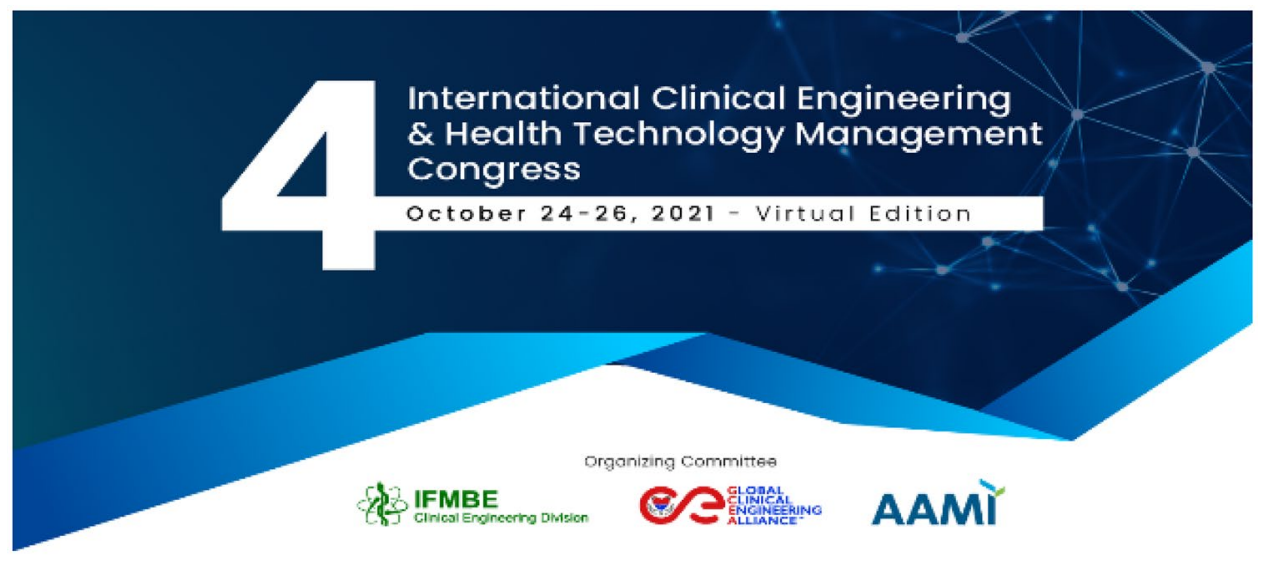

Why am I thrilled about Global Clinical Engineering (CE) Day 2021 - since 2015 celebrated on October $21^{\text {st? }}$ ? And here's why should you also be excited.

It's no longer one day but now a week, October 19-26! And Clinical Engineers have gotten unprecedented recognition. This week-long festivity highlights the contribution and impact Clinical Engineers make around the world every day! Begun in China in 2015, the Global CE Day Event will again be held in China on October 23. In 2020, CED featured $22 \mathrm{~h}$ of programming, with the Olympics-style around-the-globe celebration, see https://www.globalcea.org/globalceday! See also nearly 70 countries' CE status reports prepared for Global CE Day in October 2019: https://ced.ifmbe.org/blog/ ifmbe-ced-cestatus-cesummit2019.html.

Clinical Engineering practitioners around the world ( 800,000 plus in 130 countries according to the World Health Organization-WHO's 2018 survey) have rallied around the profession locally and globally during COVID19 in 2020-2021 to make a huge difference in healthcare delivery. At the last two WHO World Health

Tom Judd

1 Global Clinical, Engineering Alliance, 8371 SE 57th ST, WA 98040 Mercer Island, United States
Assemblies, LMIC government leaders have pointed to Ventilators and Oxygen as national priorities. WHO now classifies PPE, all kinds of oxygen systems, and digital health tools as medical equipment alongside traditional devices! With WHO in 2020-now, we have held over 40 $\mathrm{CE}$ and COVID19-related educational webinars, with registrants from 170 countries.

This year we will hold our biannual Congress $\left(4^{\text {th }}\right.$ ICEHTMC) during the Week on October 23-26. It will be Virtual on the Zoom Events platform (with Zoom Health a key sponsor) with 1500 colleagues from 120 countries expected. After three previous Congresses, China (2015), Brazil (2017) and Rome (2019 - 1000 attendees from 70 countries), in 2021 , we will have a record-breaking $200+$ presentations from colleagues in 70 countries, selected from 400 abstracts with 700 co-authors. 2021 Keynotes will be: WHO \& the Global CE Community; Global CE Credentialing; COVID Global CE Grand Challenges; and Why GCEA?

CE's global footprint and influence has grown. CED's Board \& Collaborator team has grown to over 420 colleagues from 165 countries, notably due to our global work with COVID19. At WHO's urging, CED not only joined the global effort, but started in March 2020 a weekday 
e-newsletter offering vetted articles on COVID19 topics. From these 'Hacking COVID19' newsletters, there are now over 2000 items available at https://www.globalcea.org/hcu. In October, we will surpass 400 weekday issues that have been sent daily to 13,000 global subscribers, and various Social Media outlets.

CED and GCEA's collaboration also has aligned with several global entities of note, such as the International Hospital Federation, the Every Breath Counts Coalition, the Gates Foundation, and many other NGOs in our common efforts to ensure health technologies are appropriately selected, deployed, and sustained to improve the quality and safety of healthcare delivery around the world every day.
So, as you can see, the CE global team is now a "Community". Via a WhatsApp group, we now have daily discussions with 200 colleagues from all over the world bridging the gap of ideas and practices across cultures. We have seen Global CE Day grow in scope and significance. Why? Because the use of health technologies has never been greater and therefore the need for local and global clinical engineering expertise continues to expand. Join us as we move forward!

Publisher's Note Springer Nature remains neutral with regard to jurisdictional claims in published maps and institutional affiliations. 